\title{
Scientific Program (updated to July 15, 2009)
}

\author{
Thursday, September 17, 2009
}

$18^{\text {th }}$ Advanced Course "NEW INSIGHTS IN WHITE MATTER DISEASES"

Lecture Hall “TERPSICHORE” (Diagnostic)

\author{
Chairpersons : Olof Flodmark (Sweden), Stephanos Lahanis (Greece) \\ 08:30-09:00 Myelin maturation and white matter development: an embryologic eye view. \\ John Hesselink (USA) \\ 09:00-09:30 Concepts of myelin and myelination in MRI \\ John Hesselink (USA) \\ 09:30-10:00 Conventional, diffusion and magnetization transfer MRI in the study of myelination. \\ Maria Argyropoulou (Greece) \\ 10:00-10:30 White-matter diseases: radiologic-pathologic correlation \\ James Smirniotopolous (USA) \\ 10:30-11:00 Coffee Break \\ Chairpersons : John Hesselink (USA), Clementine Karageorgiou (Greece) \\ 11:00-11:30 New clinicopathological perspectives in MS \\ Ludwig Kappos (Switzerland) \\ 11:30-12:00 New diagnostic criteria in MS \\ Frederik Barkhof (The Netherlands) \\ 12:00-12:30 Monitoring therapy in MS \\ Ernst Wilhelm Radue (Switzerland) \\ 12:30-13:00 White matter lesions in ageing and dementia \\ Frederik Barkhof (The Netherlands) \\ 13:00-14:30 Lunch \\ Chairpersons : Pia Sundgren (Sweden), Frederik Barkhof (The Netherlands) \\ 14:30-15:00 Therapeutic effects on white matter \\ Alex Rovira (Spain) \\ 15:00-15:30 MRI pattern recognition in white matter disorders \\ MarjoVan der Knaap (The Netherlands) \\ 15:30-16:00 Coffee Break \\ Chairpersons : Pia Sundgren (Sweden), Frederik Barkhof (The Netherlands) \\ 16:00-16:30 MRI definition of novel white matter disorders. \\ MarjoVan der Knaap (The Netherlands) \\ 16:30-17:00 Differential diagnosis of white matter lesions at high-field MR. \\ Majda Thurnher (Austria) \\ 17:00-17:30 Parcelation of the white matter using DTI: insights into the functional connectivity of the brain \\ Spyridon Kollias (Switzerland) \\ 17:30-18:00 3-Testa study of the spinal cord white matter \\ Luca Albini Riccioli (Italy) \\ 19:00 \\ WELCOME RECEPTION at Hilton Galaxy Terrace
}


Thursday, September 17, 2009

$2^{\text {nd }}$ Advanced Interventional "SPINE AND SPINAL CORD"

Lecture Hall “HESPERIDES” (Interventional)

08.30-10.30 SPINAL VASCULAR MALFORMATIONS

Chairpersons : Luc Picard (France), Anton Valavanis (Switzerland)

08:30-9:00 Classification of spinal cord arteriovenous malformations

Timo Krings (Canada)

09:00-9:30 Imaging of the spinal vascular malformations

Robert Nijenhuis (The Netherlands)

09:30-10:00 Intrinsic spinal cord AVMs

Georges Rodesch (France)

10:00-10:30 Dural AVMs of the spinal cord

Anton Valavanis (Switzerland)

10:30-11:00 Coffee Break

11.00-17.30 SPINE TREATMENTS ACROSS EUROPE

Chairpersons : Jean Baptiste Martin (Switzerland), Spyridon Kavvadias (Greece)

11:00-11:30 Tumor ablation of the spine

Afshin Gangi (France)

11:30-12:00 Disc herniation: diagnostic procedures

Alexis Kelekis (Greece)

12:00-12:30 Disc herniation: therapeutic procedures

Cosma Andreula (Italy)

12:30-13:00 Disc ozone therapy: does it replace surgery?

Mario Muto (Italy)

13:00-14:30 Lunch

Chairpersons : Jacques Chiras (France), Charalambos Drossos (Greece)

14:30-15:00 Advances in percutaneous treatment of low back pain

Jacques Theron (France)

15:00-15:30 Percutaneous treatment of the cervical spine

Giovanni Carlo Anselmetti (Italy)

15:30-16:00 Coffee Break

Chairpersons: Jacques Chiras (France), Charalambos Drossos (Greece)

16:00-16:30 Kyphoplasty. Better or worse than vertebroplasty?

Kai Wilhelm (Germany)

16:30-17:00 Vertebroplasty. What's new?

Hendrik Fransen (Belgium)

17:00-17:30 Treatments for sciatica mimics: facets and sacroiliac joints

Massimo Gallucci (Italy)

19:00

WELCOME RECEPTION at Hilton Galaxy Terrace 
Friday, September 18, 2009

Morning

Lecture Hall “TERPSICHORE"

08:00-09:15 REFRESHER COURSE D I: The brain movement disorders

Chairpersons : Jordi Ruscalleda (Spain), Panayotis Toulas (Greece)

MRI biomarkers of Parkinson disease and Parkinsonian disorders: State of the art

Maria Grazia Bruzzone (Italy)

Neuroimaging challenges in abnormal movement diseases

Beatriz M. Gomez-Anson (Spain)

Present role of nuclear medicine in the diagnostic work-up of cognitive impairement in Lewy body disease Zuzane Walker (United Kingdom)

When and how should patients with movement disorders be imaged, if at all

Tarek Yousry (United Kingdom)

09:15-10:30 OPENING CEREMONY

ESNR Awards

European Society of Neuroradiology: 40 years. A long way towards maturity

Luc Picard (France)

10:30-11:00 Coffee Break

11:00-11:25 STATE OF THE ART LECTURE

Chairpersons : Athanassios D. Gouliamos (Greece)

11.00-11.25 Neuroradiology between Hippocratic encephalocentrism and Aristotelian cardiocentrism Anton Valavanis (Switzerland)

11:25-13:00 LECTURE AND SCIENTIFIC PAPERS

Chairpersons : Guido Wilms (Belgium), Aggelik Tavernaraki (Greece)

11:25-11:50 Low grade gliomas; can we predict tumor behaviour from imaging features?

Charles Romanowski (United Kingdom)

11:50-13:00 Scientific Papers 1 (TE1): Imaging of brain tumors-multimodality approach I

13:00-14:00 Lunch

\section{Afternoon}

14:00-14:25 LECTURE

Chairpersons : Athanasios Dimitriadis (Greece)

14:00-14:25 Radiologic grading of Astrocytoma

James Smirniotopolous (USA)

14:25-16:00 LECTURE AND SCIENTIFIC PAPERS

Chairpersons : Dimitrios Vasilopoulos (Greece), Goraj Bozena (The Netherlands)

14:25-14:50 Present role of nuclear medicine in the diagnostic work-up of brain tumors

Klaus Tatsch (Germany) 
14:50-16:00 Scientific Papers 2 (TE2): Inflammatory and demyelinating diseases of the brain I

16:00-16:30 Coffee Break

16:30-17:45 LECTURE AND SCIENTIFIC PAPERS

Chairpersons : Jim Smirniotopoulos (USA), Jerzy Walecki (Poland)

16:30-16:55 Neuroimaging contributes to the diagnosis, management and prognosis of patients with craniocerebral trauma

Paul Parizel (Belgium)

16:55-17:45 Scientific Papers 3 (TE3): Imaging in epilepsy

17:45-18:45 Case based review session: The brain

Anne Osborn (USA) 
Friday, September 18, 2009

\title{
Morning
}

08:00-09:15 REFRESHER COURSE INT 1: Aneurysm treatment by modelling of flow

Chairpersons : Marco Leonardi (Italy), James Byrne (UK)

Potentials of flow modelling in cerebral aneurysms

Daniel Rufenacht (Switzerland)

Flow diversion for the treatment of complex cerebral aneurysms

Jacques Moret (France)

Different flow diversion concepts in the endovascular aneurysms treatment

Saruhan Cekirge (Turkey)

Pipeline embolization device for the treatment of cerebral aneurysms

Istvan Szikora (Hungary)

10:30-11:00 Coffee Break

11:25-13:00 LECTURE AND SCIENTIFIC PAPERS

Chairpersons : Luc Picard (France), Panayotis Vlahopoulos (Greece)

11:25-11:50 The possible use of flow diverting stens in the treatment of large-giant cerebral aneurysms Marco Leonardi (Italy)

11:50-13:00 Scientific Papers 4 (HE1) : Interventional-aneurysms

13:00-14:00 Lunch

13:00-14:00 SATELLITE SESSIONS

\author{
Afternoon \\ 14:00-16:00 LECTURES AND SCIENTIFIC PAPERS \\ Chairpersons : Rudiger von Kummer (Germany), Menno Sluzewski (The Netherlands) \\ 14:00-14:25 Hemorrhagic intracranial dissections \\ Serge Bracard (France) \\ 14:25-14:50 Treatment of wide neck aneurysms with balloon remodelling \\ René Chapot (France) \\ 14:50-16:00 Scientific Papers 5 (HE2): Interventional—aneurysms and AVMs \\ 16:00-16:30 Coffee Break \\ 16:30-17:45 LECTURE AND SCIENTIFIC PAPERS \\ Chairpersons : Anton Valavanis (Switzerland), Dimitrios Rologis (Greece) \\ 16:30-16:55 The unruptured aneurysm controversy \\ Andrew Molyneux (United Kingdom) \\ 16:55-17:45 Scientific Papers 6 (HE3) : Interventional-stroke I
}


Friday, September 18, 2009

\section{Morning}

Lecture Hall "THALIA"

Chairpersons : Nuno Canto-Moreira (Portugal), George Karas (The Netherlands)

11:50-13:00 Scientific Papers 16 (TH1): Inflammatory and demyelinating diseases of the brain II

13:00-14:00 Lunch

\section{Afternoon}

Chairpersons : Sotiris Giouroukos (Greece), P. Krupa (Czech Republic)

14:50-16:00 Scientific Papers 17 (TH2): Pediatric neuroradiology I

16:00-16:30 Coffee Break

Chairpersons : Gaida Krumina (Latvia)

16:55-17:45 Scientific Papers 18 (TH3): Demyelinating diseases III 
Saturday, September 19, 2009

Morning

Lecture Hall “TERPSICHORE"

08:15-09:45 REFRESHER COURSE D II

Chairpersons : E. Turgut Tali (Turkey), Nickolas Papanikolaou (Greece)

New imaging modalities in clinical practice. The top 5 clinical questions answered by:

Spectroscopy

Efstathios Gotsis (Greece)

DWI

Majda Thurnher (Austria)

PWI

Paul Parizel (Belgium)

DTI

Stefan Sunaert (Belgium)

f-MRI

Spyridon Kollias (Switzerland)

09:45-10:15 LECTURE

Chairpersons : Kyriakos Strigaris (Greece)

09:45-10:15 CNS involvement in autoimmune diseases

Nicholas Patronas (USA)

10:15-10:45 Coffee Break

10:45-12:10 LECTURE AND SCIENTIFIC PAPERS

Chairpersons : Danielle Balériaux (Belgium), Eftychia Kapsalaki (Greece)

10:45-11:10 Low back pain. Use or abuse of advanced imaging?

Spyros Karampekios (Greece)

11:10-12:10 Scientific Papers 7 (TE4): Imaging of the spine and spinal cord

12:10-13:15 REFRESHER COURSE D III: Pediatric neuroradiology

Chairpersons : Paolo Tortori Donati (Italy), Ioannis Nikas (Greece)

Training in fetal MRI-where we stand, where we should go

Daniela Prayer (Austria)

MR advances in perinatal tensor diffusion imaging

Guy Sebag (France)

Congenital and acquired pediatric cerebrovascular disorders

Andrea Rossi (Italy)

13:15-14:15 ESNR GENERAL ASSEMBLY

14:15-15:00 Lunch

14:15-15:00 SATELLITE SESSIONS

\author{
Afternoon \\ 15:00-16:50 LECTURE AND SCIENTIFIC PAPERS \\ Chairpersons : Stefan Sunaert (Belgium), Efrosini Z. Papadaki (Greece)
}


15:00-15:25 Neuroradiology in UEMS - a new professional development in Europe

Olof Flodmark (Sweden)

15:25-16:50 Scientific Papers 8 (TE5): Advanced imaging modalities I

16:50-17:15 Coffee Break

17:15-18:30 LECTURE AND SCIENTIFIC PAPERS

Chairpersons : Paul Parizel (Belgium), Ioannis Andreou (Greece)

17:15-17:40 Viral-Prion diseases of the CNS

Turgut Tali (Turkey)

17:40-18:30 Scientific Papers 9 (TE6): Free topics

18:30-19:30 Case based review session: The Spine

Johan van Goethem (Belgium)

21:30

GALA DINNER 
Saturday, September 19, 2009

Morning

08:15-09:25 REFRESHER COURSE INT II: Endovascular treatment of acute stroke

Chairpersons : Claude Manelfe (France), E. Arhodakis (Greece)

Acute stroke. Diagnostic assessment of the cerebral thrombolysis candidate

Catherine Oppenheim (France)

Intra-arterial thrombolysis in acute ischemic stroke

Rudiger Von Kummer (Germany)

Mechanical thrombectomy for revascularization in acute ischemic stroke

Martin Schumacher (Germany)

09:25-10:15 LECTURES

Chairpersons : Augusto Goulão (Portugal), Georges Rodesch (France)

09:25-09:50 Angioarchitectural analysis and treatment strategy in BAVMs

George Magoufis (Greece)

09:50-10:15 Treatment of BAVMs. Case selection for glue vs Onyx.

James Byrne (United Kingdom)

10:15-10:45 Coffee Break

10:45-13:15 LECTURE AND SCIENTIFIC PAPERS

Chairpersons : Martin Schumacher (Germany), Achilleas Chatziioannou (Greece)

10:45-11:10 Endovascular treatment of intracranial atherosclerotic disease Juergen Reul (Germany)

11:10-13:15 Scientific Papers 10 (HE4): Interventional stroke II

14:15-15:00 Lunch

14:15-15:00 SATELLITE SESSIONS

\section{Afternoon}

15:00-16:50 LECTURE AND SCIENTIFIC PAPERS

Chairpersons : Pedro Vilela (Portugal), Karl Olof Lovblad (Switzerland)

15:00-15:25 Stroke in the young adult etiology and imaging

W. K. Kling Chong (United Kingdom)

15:25-16:50 Scientific Papers 11 (HE5): Cerebrovascular disease imaging I

16:50-17:15 Coffee Break

17:15-18:30 LECTURE AND SCIENTIFIC PAPERS

Chairpersons : Marek Sasiadek (Poland), Zulejha Merhemic (Bosnia \& Herzegowina)

17:15-17:40 Sellar and parasellar lesions

Antonios Drevelegas (Greece)

17:40-18:30 Scientific Papers 12 (HE6): Pediatric neuroradiology II 
Saturday, September 19, 2009

\section{Morning}

Lecture Hall "THALIA"

Chairpersons : Chrysoula Samara (Greece), V. Katsaros (Greece)

11:50-12:10 Scientific Papers 19 (TH4): Imaging of brain tumors-multimodality approach II

\section{Afternoon}

Chairpersons : Catherine Oppenheimer (France), Majda Thurnher (Austria)

15:25-16:50 Scientific Papers 20 (TH5): Cerebrovascular disease imaging II 
Sunday, September 20, 2009

Morning

Lecture Hall “TERPSICHORE"

08:15-09:30 REFRESHER COURSE D IV: Epilepsy

Chairpersons : Peter Barsi (Hungary), Damianos E. Sakas (Greece)

MR imaging in the presurgical workup of patients with drug resistant epilepsy

Matilda Papathanasiou (Greece)

Novel contrasts to reveal more about the brain in epilepsy

Fergus J. Rugg-Gunn (United Kingdom)

Functional neuroimaging in the preoperative evaluation of language indrug-resistant epilepsy

Elio Maccagnano (Italy)

09:30-10:00 LECTURE

Chairperson : Lambros Vlahos (Greece)

09:30-10:00 Hearning loss; imaging of the auditory pathway

Jan Casselman (Belgium)

10:00-10:30 Coffee Break

10:30-12:00 LECTURE AND SCIENTIFIC PAPERS

Chairpersons : Tatjana Stosic Opincal (Serbia), Nikos Bontozoglou (Greece)

10:30-10:55 Paradign shift in CSF circulation: CSF absorption by brain capillaries instead of arachnoid granulations

Dan Greitz (Sweden)

10:55-12:00 Scientific Papers 13 (TE7): Head and Neck imaging and intervention

12:30-13:30 CLOSING REMARKS 
Sunday, September 20, 2009

Morning

Lecture Hall “HESPERIDES”

8:30-10:00 Scientific Papers 14 (HE7) : Spinal interventions

Chairpersons : Johan van Goethem (Belgium)

10:00-10:30 Coffee Break

10:30-12:30 LECTURES AND SCIENTIFIC PAPERS

Chairpersons : Anton Valavanis (Switzerland), Spyridon Kollias (Switzerland)

10:30-10:55 Intracranial DAVMs. Classification, natural history and treatment planning Georges Rodesch (France)

10:55-11:20 Transarterial Onyx embolization for the treatment of intracranial DAVMs Christophe Cognard (France)

11:20-12:30 Scientific Papers 15 (HE8): Advanced imaging modalities II f-MRI, DTI 